\title{
Knowledge and Attitude Towards COVID-19 and Its Associated Factors Among Health Care Providers in Ethiopia: A Systematic and Meta-Analysis
}

\author{
Maru Meseret*, Zegeye Regasa, Gizaw Hailye, Bayou Tilahun, Amare Dagnew \\ Department of Health Informatics, College of Health Science, Debre Markos University, Debre Markos, Ethiopia \\ Email address: \\ marumeseret@gmail.com (M. Meseret), zegeyerega@gmail.com (Z. Regasa), ghailiye463@gmail.com (G. Hailye), \\ bayutilahun5@gmail.com (B. Tilahun) \\ ${ }^{*}$ Corresponding author
}

\section{To cite this article:}

Maru Meseret, Zegeye Regasa, Gizaw Hailye, Bayou Tilahun, Amare Dagnew. Knowledge and Attitude Towards COVID-19 and Its Associated Factors Among Health Care Providers in Ethiopia: A Systematic and Meta-Analysis. American Journal of Clinical and Experimental Medicine. Vol. 9, No. 4, 2021, pp. 109-121. doi: 10.11648/j.ajcem.20210904.14

Received: June 30, 2021; Accepted: July 15, 2021; Published: August 6, 2021

\begin{abstract}
Objective: The main aim of this study is to determine the pooled estimate of good knowledge and positive attitude towards COVID-19 among health care providers in Ethiopia. Methods: This review was registered on PROSPERO with registration number CRD42020213000. PubMed, Advanced Google search, and Google Scholar databases were searched up to June 20, 2020, to identify relevant articles. Result: This research searched a total of, 126 articles, from this articles 19 from PubMed, 6 from advanced Google search, 45 from Google scholar databases, and 56 were pre-print unpublished sources. A total of 3752 study participants within 10 articles were used to pool the prevalence of Knowledge and attitude towards COVID-19 among health care providers in Ethiopia and the pooled prevalence of knowledge and attitude towards COVID-19 was $73.01 \%$ with $95 \% \mathrm{CI}(65.8 \%-80.21 \%)$ and $70.72 \%(59.68 \%-81.77 \%)$ respectively. Place of residence $[\mathrm{AOR}=0.24,95 \%$ CI (0.14-0.43)], and social media use [AOR $=2.51,95 \%$ CI (1.53-4.12)] for knowledge while educational status [AOR $=2.41$, 95\% CI (1.33-4.39)], and status of knowledge were also identified as factors affecting attitude towards COVID 19 among healthcare providers in Ethiopia. Conclusion: The level of knowledge and attitude towards COVID 19 among healthcare providers working in Ethiopia was very low and variables such as residence and social media use were factors affecting knowledge while educational status and knowledge were factors affecting attitude towards COVID 19 among healthcare providers.
\end{abstract}

Keywords: COVID-19, Knowledge, Attitude, Health Care Providers, Ethiopia

\section{Introduction}

\subsection{Background}

Bad news all over the world was disseminated in late December 2019 from Wuhan, Hubei Province, China reporting a group of pneumonia cases of unknown causes. On 9 January 2020, China CDC reported a novel coronavirus as the causative agent of this outbreak, later named coronavirus disease 2019 (COVID-19) [1].

Coronavirus is one of the many viruses existing worldwide and it can be transmitted via birds and mammals. Human beings as being mammals are susceptible to infection and transmission of the virus [2, 3]. This virus is currently recognized to cause respiratory illnesses ranging from the mild common cold to severe acute respiratory syndrome (SARS) [4].

Regarding mode of transmission, this virus can infect humans by coming into close contact (about 6 feet or two arm lengths) with a person who has COVID-19 through respiratory droplets when an infected person coughs, sneezes, or talks. It may also able to get into by touching a surface or object that has a virus on it [5]. Human beings can get protected from this virus by avoiding being exposed to the virus using strategies such as stay at home as much as possible, physical distancing, wearing personal protective 
equipment (PPE), cleaning and disinfecting frequently touched surfaces, and washing hands often with soap and water for at least 20 seconds, or use an alcohol-based hand sanitizer that contains at least $60 \%$ alcohol $[5,6]$.

Everyone is at risk of acquiring COVID-19 [6] however, people most at risk of acquiring the disease are those who are in contact with or care for patients with COVID-19. This inevitably places health care workers (HCWs) at high risk of infection. Protecting $\mathrm{HCWs}$ is of paramount importance to the world health organization (WHO) [7]. Health care workers' knowledge and attitude towards COVID-19 infection prevention and control also play a significant role in line with efforts that would be taken by WHO to protect HCWs [7].

Expecting a positive attitude towards COVID-19 without having sufficient knowledge is like waiting for an egg while there is no chicken. Researches from Asian countries such as China, Vietnam, and India indicated a high number of health care workers had good knowledge and attitude towards COVID-19 which ranges from $86 \%$ to $89 \%$ for good knowledge and from $85 \%-94 \%$ for positive attitude [8-10]. However, a lower level of good knowledge was also observed from the other study in India [11].

A slightly lower level of knowledge ranging from $69 \%$ to $87 \%$ and attitude which ranges from $80-86 \%$ was documented by research from the Middle East Asian countries such as Saudi Arabia [12], United Arab Emirates (UAE) [13], and Yemen [14]. Literature regarding, the level of knowledge and attitude towards COVID-19 in African countries was also assessed and the good knowledge varies from $62 \%$ to $70 \%$ while the level of positive attitude was $21 \%$ in Ghana [15] and Uganda [16].

Inconsistent findings towards good knowledge and positive attitude were also observed in Ethiopia which ranged from $53.2 \%$ to $88.2 \%$ for good knowledge [17-26] and from $56.6 \%$ to $89.8 \%$ for attitude $[17,19,21,23-26]$ towards COVID-19.

\subsection{The Rationale of the Review}

In Ethiopia, up to 18 October 2020, a total of 1,397,348 was screened for COVID-19, and 89, 137 of them were confirmed COVID-19 cases with a fatality rate of 15 deaths per 1000 population. Health care providers are at the highest risk of acquiring the infection [27-29]. Understanding the natural history of the disease and healthcare workers being ready and interested to prevent themselves from exposure would largely reduce the rate of infection. Here, having knowledge and attitude towards the disease in question plays a critical role. Findings in Ethiopia indicated inconsistent findings against knowledge and attitude towards COVID 19 among healthcare providers. Bringing inconsistent findings to a pooled estimate would narrow this gap.

Lately, we found that a systematic and meta-analysis had been conducted and published in Ethiopia [30] but failed to include factor analysis. Therefore, the main aim of this study was to determine the pooled estimate of good knowledge and positive attitude and its associated factors towards COVID-
19 among health care providers in Ethiopia.

\section{Methods}

\subsection{Protocol Registration}

This review was conducted according to a priori published protocol on PROSPERO International Prospective Register of systematic reviews for publication registration number CRD42020213000 (available at https://www.crd.york.ac.uk/PROSPERO/\#myprospero).

Literature search: Initially, databases were searched for the same systematic review done before to avoid duplications of efforts. Boolean operators were used for searching studies. PubMed, Advanced Google search, and Google Scholar databases were searched up to 18 October 2020 to identify relevant articles. The keywords and medical subject headings ((((Knowledge) AND/OR (Attitude)) AND (COVID 19)) AND (Healthcare providers)) AND (Ethiopia) were used to screen for potentially relevant studies.

\subsection{Eligibility Criteria}

Studies were eligible for inclusion in the review if they met the following criteria: (1) published in the English language; (2) study participants were healthcare providers in Ethiopia; (3) studies which reported sufficient knowledge and attitude towards COVID 19: Studies of all design. Studies were excluded if they met the following criteria: (1) not an original study, such as a review paper (2) Studies difficult to access full text and studies which didn't report specific outcomes knowledge and attitude were excluded.

\subsection{Patient and Public Involvement}

No patient involved

\subsection{Data Extraction and Study Quality Assessment}

Data from eligible studies were extracted independently by two reviewers (Maru and Zegeye). The following data were extracted from all studies: author, study area (region), study setting, study year, publication year, and sample size, number of participants, study design, sampling technique, and prevalence. Disagreements between the two reviewers during data extraction were reconciled by a 3rd investigator (Gizaw). One author (Bayou) assessed the quality of the articles based on the Joanna Briggs Institute Meta-Analysis of Statistics Assessment and Review Instrument (JBIMAStARI) adapted for both cross-sectional/case-control study design was used [31]. Disagreement raised by two quality assessors was repeated and further solved with the involvement of the third reviewer.

\subsection{Data Synthesis and Statistical Analysis}

STATA 14 statistical software was used for meta-analysis. The existence of heterogeneity was assessed by testing publication bias using funnel plot and more objectively by Egger's regression test. I-squared statistics were used to 
check the heterogeneity of the studies. The random-effects model was employed to estimate the overall prevalence. Subgroup analysis based on the study area (region), number of study participants, and study year was conducted to see the variation in outcomes.

\section{Result}

\subsection{Search Results}

This research searched a total of, 126 articles, from this articles 19 from PubMed, 6 from advanced Google search, 45 from Google scholar databases, and 56 were pre-print unpublished sources. Thirty eight studies title and abstract was irrelevant to the study, 78 studies were from countries other than Ethiopia and therefore removed from the study (Figure 1).

\subsection{Characteristics of Studies}

A total of 10 studies were included in the analysis and 7 $(70 \%)$ of them were institutional based, $1(10 \%)$ community based and $2(20 \%)$ online cross-sectional studies involving simple random sampling (6 studies), census (1 study), convenience (3 studies) sampling techniques. Nine (90\%) studies were conducted during 2020 while 1 (10\%) was done during 2019. Out of all studies included in the meta-analysis, Amhara region accounts $6(60 \%)$ (Table 1).

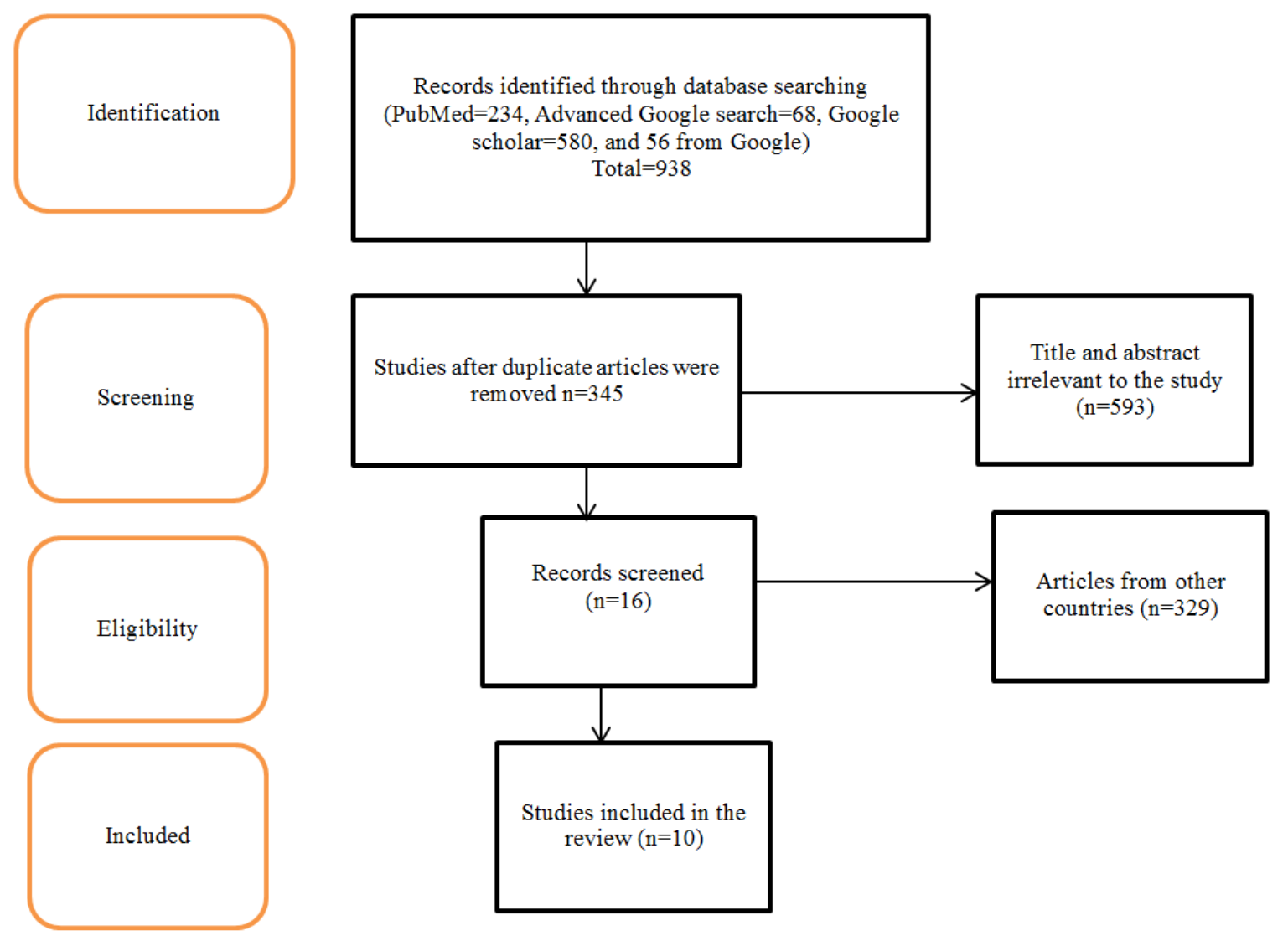

Figure 1. PRISMA flow-chart diagram describing the selection of studies.

Table 1. Characteristic of included studies in systematic review and meta-analysis.

\begin{tabular}{|c|c|c|c|c|c|c|c|c|c|}
\hline $\begin{array}{l}\text { Ser. } \\
\text { No }\end{array}$ & $\begin{array}{l}\text { First author and study } \\
\text { year }\end{array}$ & Region & Study design & $\begin{array}{l}\text { Sampling } \\
\text { technique }\end{array}$ & Study population & $\begin{array}{l}\text { Participan } \\
\text { ts }\end{array}$ & $\begin{array}{l}\text { Knowledge } \\
\text { Level (\%) }\end{array}$ & $\begin{array}{l}\text { Attitude } \\
\text { Level (\%) }\end{array}$ & $\begin{array}{l}\text { Refere } \\
\text { nce }\end{array}$ \\
\hline 1 & Abay et al, 2020 & Amhara & Cross-sectional & SRS & Health care providers & 408 & 69.6 & 56.6 & [19] \\
\hline 2 & Bedru, et al 2020 & Other & Cross-sectional & SRS & Health care providers & 397 & 88.2 & & {$[26]$} \\
\hline 3 & Belayneh et al, 2020 & Amhara & Cross-sectional & SRS & Health care providers & 408 & 73.8 & 65.7 & {$[21]$} \\
\hline 4 & Degena et al, 2020 & Other & Cross-sectional & SRS & Health care providers & 415 & 74 & 72 & {$[23]$} \\
\hline 5 & Dejen et al, 2020 & Amhara & Cross-sectional & SRS & Health care providers & 166 & 84.9 & 63.3 & {$[24]$} \\
\hline 6 & Mulusew et al, 2020 & Amhara & Cross-sectional & SRS & Health care providers & 398 & 70 & & {$[20]$} \\
\hline 7 & Zelelam et al, 2020 & Other & Cross-sectional & Convenience & Health care providers & 295 & 53.2 & 89.8 & [17] \\
\hline 8 & Hailemariam et al, 2019 & Amhara & Cross-sectional & Convenience & Health care providers & 191 & 86.4 & 76.4 & {$[25]$} \\
\hline 9 & Henok et al, 2020 & Other & Cross-sectional & Census & Health care providers & 528 & 55.9 & & {$[18]$} \\
\hline 10 & Yared et al, 2020 & Amhara & Cross-sectional & Convenience & Health care providers & 546 & 73.8 & & {$[22]$} \\
\hline
\end{tabular}




\subsection{Pooled Prevalence of Knowledge and Attitude Towards COVID-19}

A total of 3752 study participants within 10 articles were used to pool the prevalence of Knowledge and attitude towards COVID-19 among health care providers in Ethiopia. Initially, a fixed-effect model was used to pool the prevalence and a higher level of heterogeneity was observed. Therefore, a random effect model was used to pool the effect size for both knowledge and attitude towards COVID-19 in Ethiopia. The pooled prevalence of knowledge towards COVID-19 was $73.01 \%$ with $95 \%$ CI $(65.8 \%$ - 80.21\%) while for attitude the pooled prevalence of attitude towards COVID-19 was $70.72 \%$ (59.68\%-81.77\%). Moreover, the forest plot for both knowledge and attitude towards COVID-19 showed that there was a high level of heterogeneity between studies indicated by $\mathrm{I} 2=96.6 \%$ and $97.1 \%$ respectively (Figures 2 and 3 ).

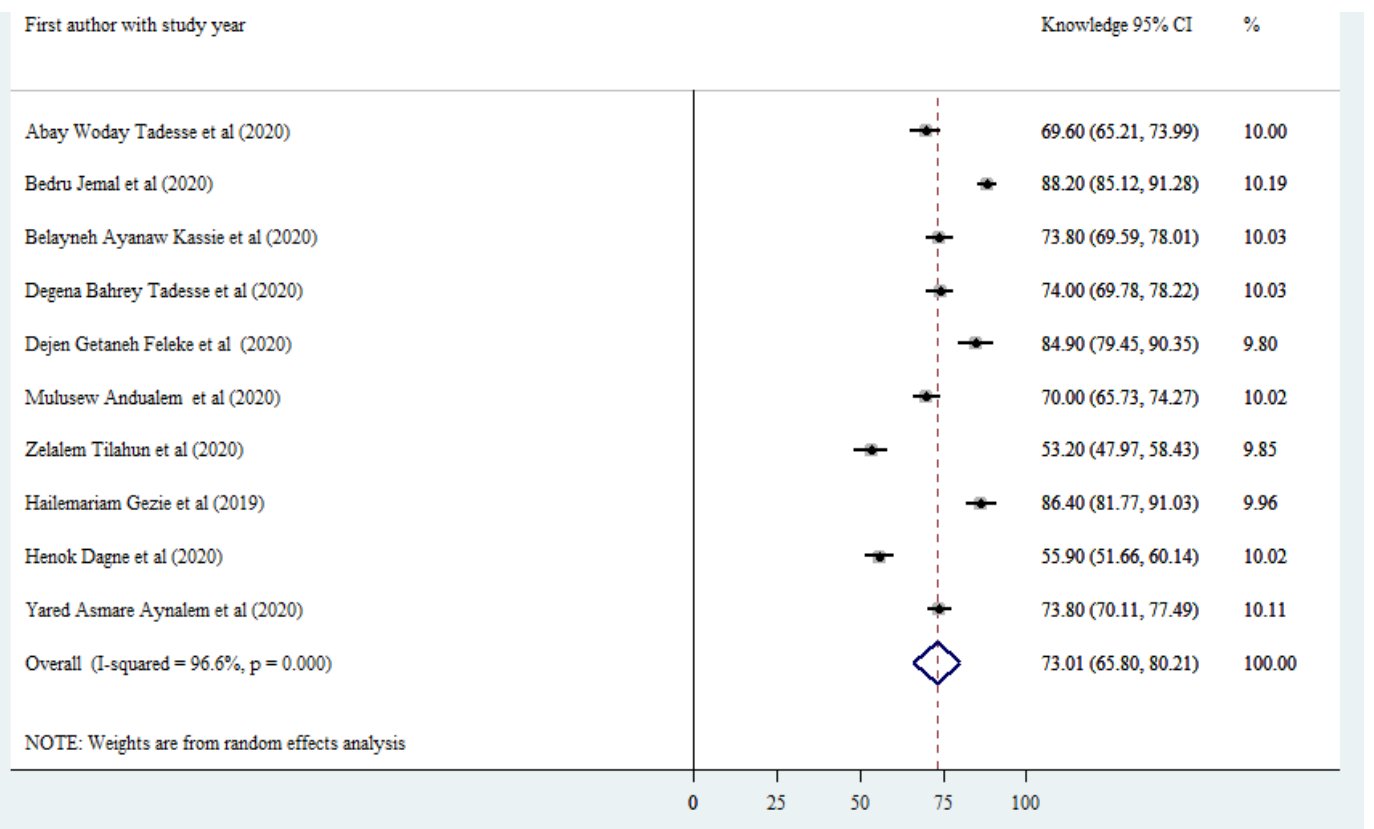

Figure 2. Forest plot showing the pooled prevalence of knowledge towards COVID-19 among health care providers in Ethiopia, 2020.

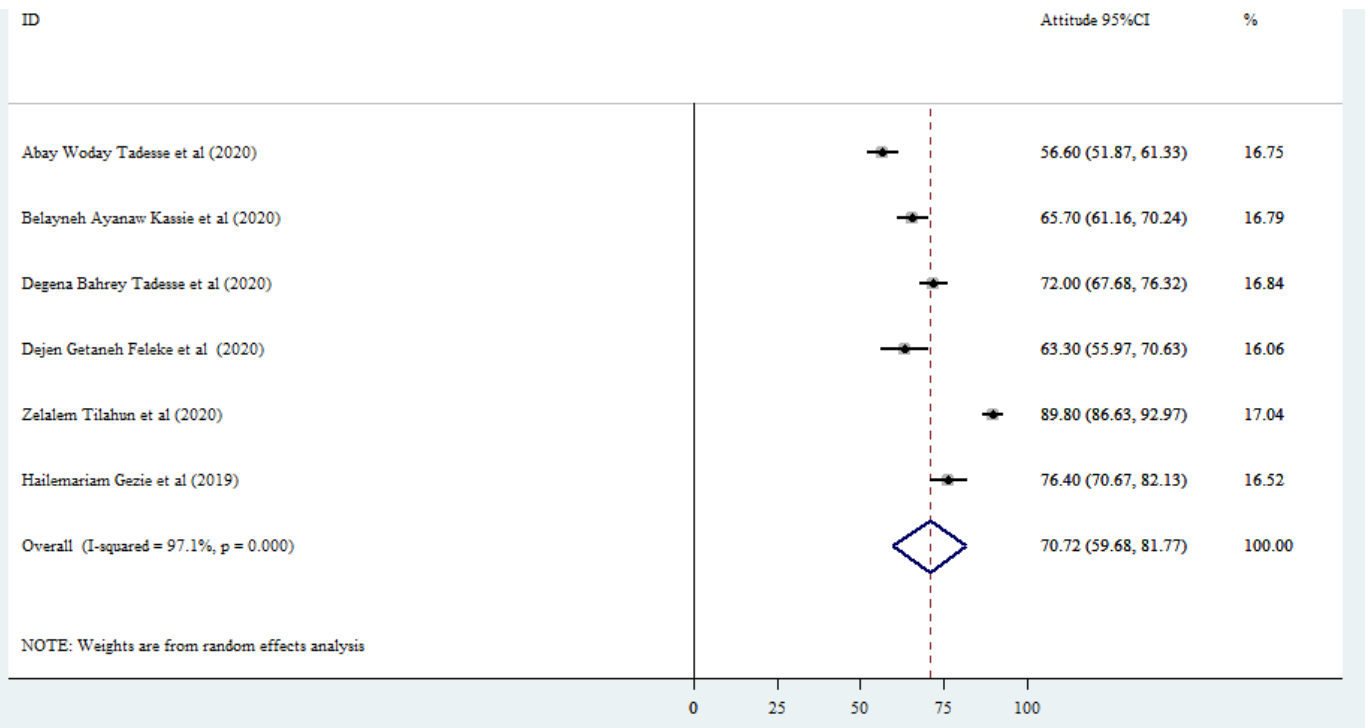

Figure 3. Forest plot showing the pooled prevalence of attitude towards COVID-19 among health care providers in Ethiopia, 2020.

\subsection{Sub-group Analysis}

This analysis tried to figure out the possible reasons for a higher level of heterogeneity between studies for both knowledge and attitude towards COVID-19. Therefore, subgroup analysis was conducted by region, publication status, and sample size for both variables. The finding showed the existence of significant association for both of the variables but all of them indicated the existence of a high level of heterogeneity suggesting that region, sample size, and study year were not the cause for heterogeneity (Figures 4, 5, 6, 7, 8 and 9). 


\section{Amhara}

Abay Woday Tadesse et al (2020)

Belayneh Ayanaw Kassie et al (2020)

Dejen Getaneh Feleke et al (2020)

Mulusew Andualem et al (2020)

Hailemariam Gezie et al (2019)

Yared Asmare Aynalem et al (2020)

Subtotal (I-squared $=89.5 \%, \mathrm{p}=0.000$ )

\section{Other}

Bedru Jemal et al (2020)

Degena Bahrey Tadesse et al (2020)

Zelalem Tilahun et al (2020)

Henok Dagne et al (2020)

Subtotal (I-squared $=98.6 \%, p=0.000$ )

Overall (I-squared $=96.6 \%, \mathrm{p}=0.000$ )

NOTE: Weights are from random effects analysis

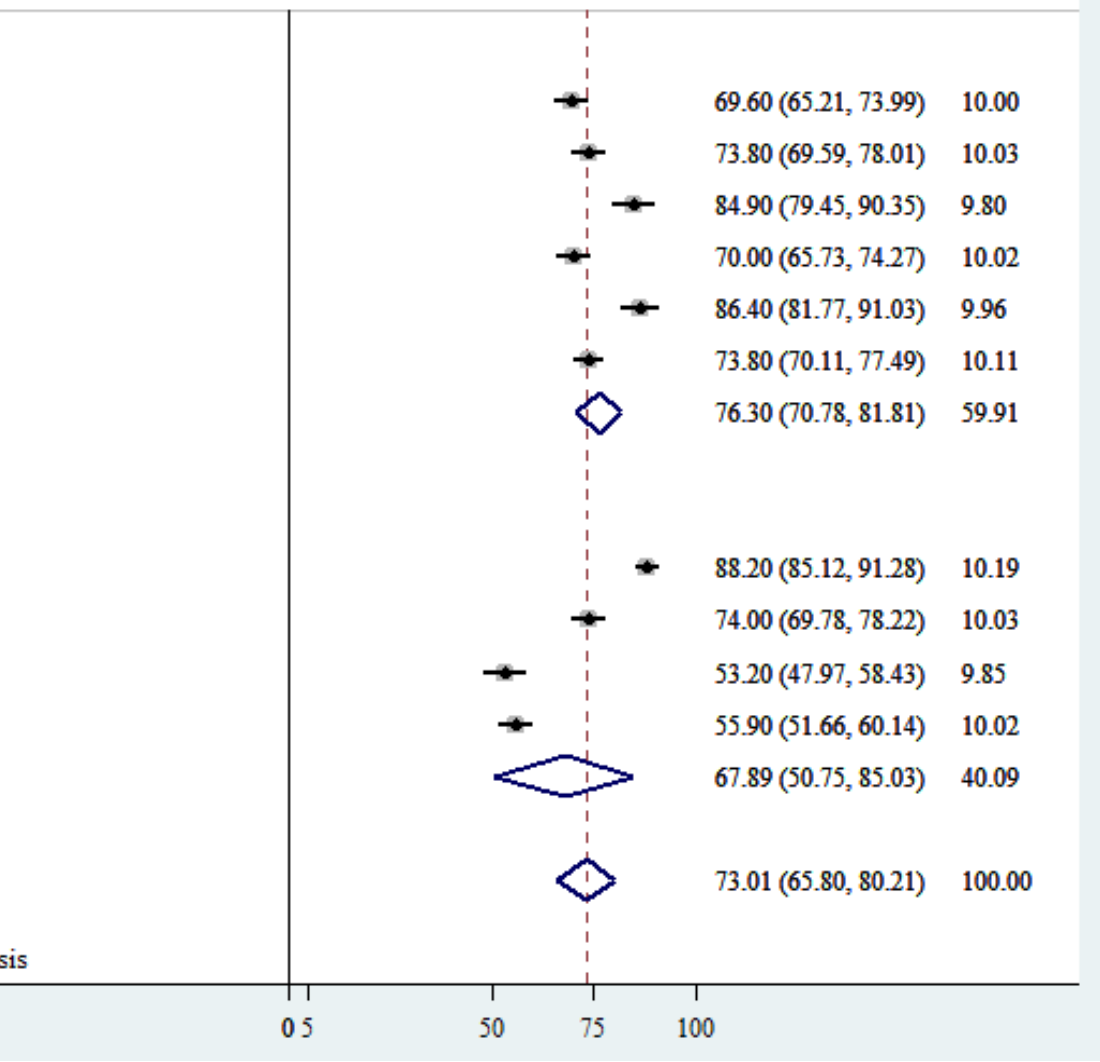

Figure 4. Sub-group analysis of knowledge by study region.

First author with study year

Attitude $95 \% \mathrm{CI} \quad \%$

Amhara

Abay Woday Tadesse et al (2020)

Belayneh Ayanaw Kassie et al (2020)

Dejen Getaneh Feleke et al (2020)

Hailemariam Gezie et al (2019)

Subtotal (I-squared $=89.1 \%, \mathrm{p}=0.000)$

Other

Degena Bahrey Tadesse et al (2020)

Zelalem Tilahun et al (2020)

Subtotal (I-squared $=97.6 \%, p=0.000)$

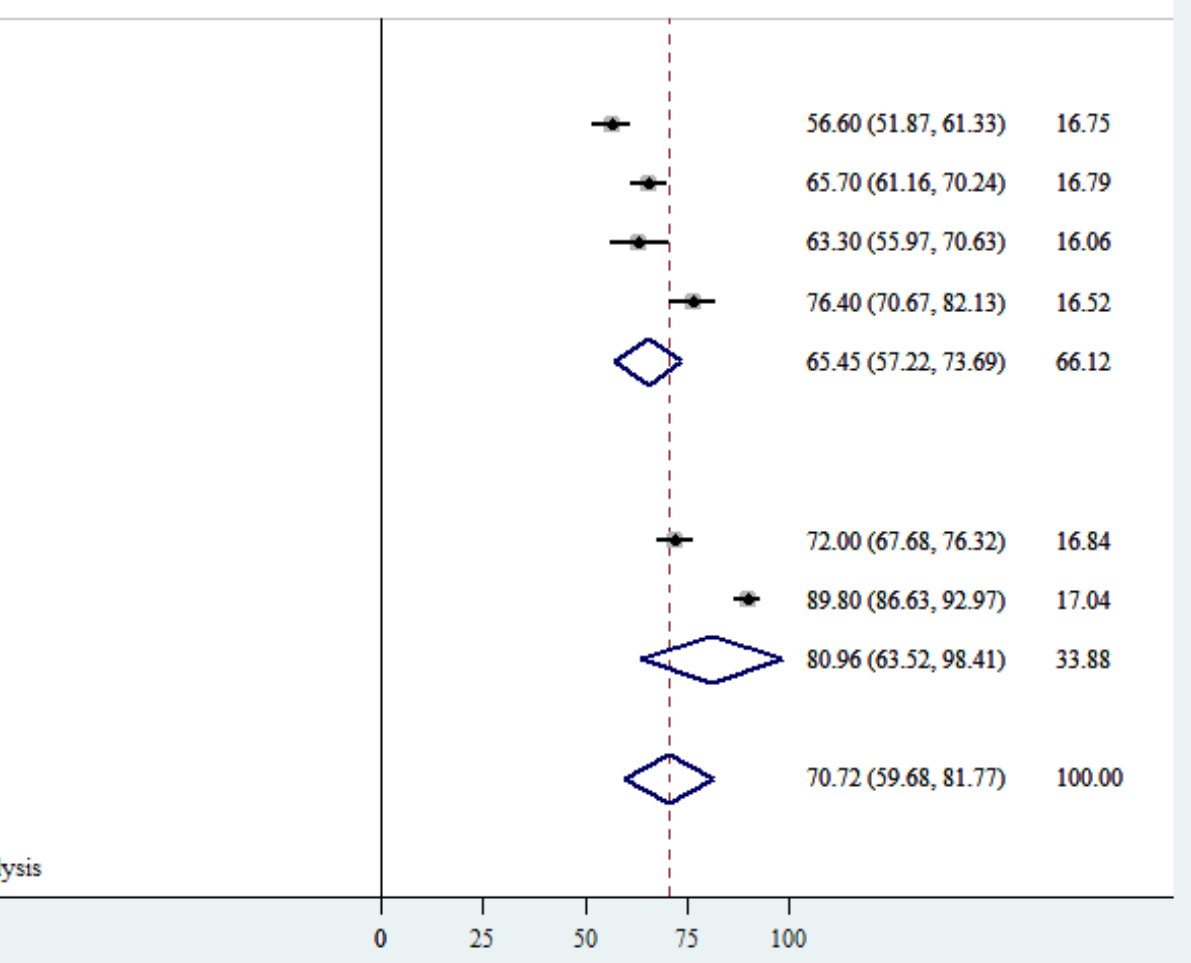

Figure 5. Sub-group analysis of attitude by study region. 
ID

\section{Preprint}

Abay Woday Tadesse et al (2020)

Bedru Jemal et al (2020)

Dejen Getaneh Feleke et al (2020)

Henok Dagne et al (2020)

Yared Asmare Aynalem et al (2020)

Subtotal (I-squared $=97.6 \%, \mathrm{p}=0.000$ )

Published

Belayneh Ayanaw Kassie et al (2020)

Degena Bahrey Tadesse et al (2020)

Mulusew Andualem et al (2020)

Zelalem Tilahun et al (2020)

Hailemariam Gezie et al (2019)

Subtotal (I-squared $=95.5 \%, \mathrm{p}=0.000$ )

Overall (I-squared $=96.6 \%, \mathrm{p}=0.000)$

NOTE: Weights are from random effects analysis
Knowledge 95\%CI \%
$69.60(65.21,73.99) \quad 10.00$

$-\quad 88.20(85.12,91.28) \quad 10.19$

$\rightarrow \quad 84.90(79.45,90.35) \quad 9.80$

$55.90(51.66,60.14) \quad 10.02$

$73.80(70.11,77.49) \quad 10.11$

$74.48(62.85,86.11) \quad 50.12$

$73.80(69.59,78.01) \quad 10.03$

$74.00(69.78,78.22) \quad 10.03$

$70.00(65.73,74.27) \quad 10.02$

$53.20(47.97,58.43) \quad 9.85$

$86.40(81.77,91.03) \quad 9.96$

$71.53(62.07,80.99) \quad 49.88$

$73.01(65.80,80.21) \quad 100.00$

Figure 6. Sub-group analysis of knowledge by publication status.

First author with study year

Attitude $95 \% \mathrm{CI} \quad \%$

Preprint

Abay Woday Tadesse et al (2020)

Dejen Getaneh Feleke et al (2020)

Subtotal (I-squared $=55.9 \%, p=0.132)$

\section{Published}

Belayneh Ayanaw Kassie et al (2020)

Degena Bahrey Tadesse et al (2020)

Zelalem Tilahun et al (2020)

Hailemariam Gezie et al (2019)

Subtotal (I-squared $=96.6 \%, p=0.000)$

Overall (I-squared $=97.1 \%, p=0.000$ )

NOTE: Weights are from random effects analysis

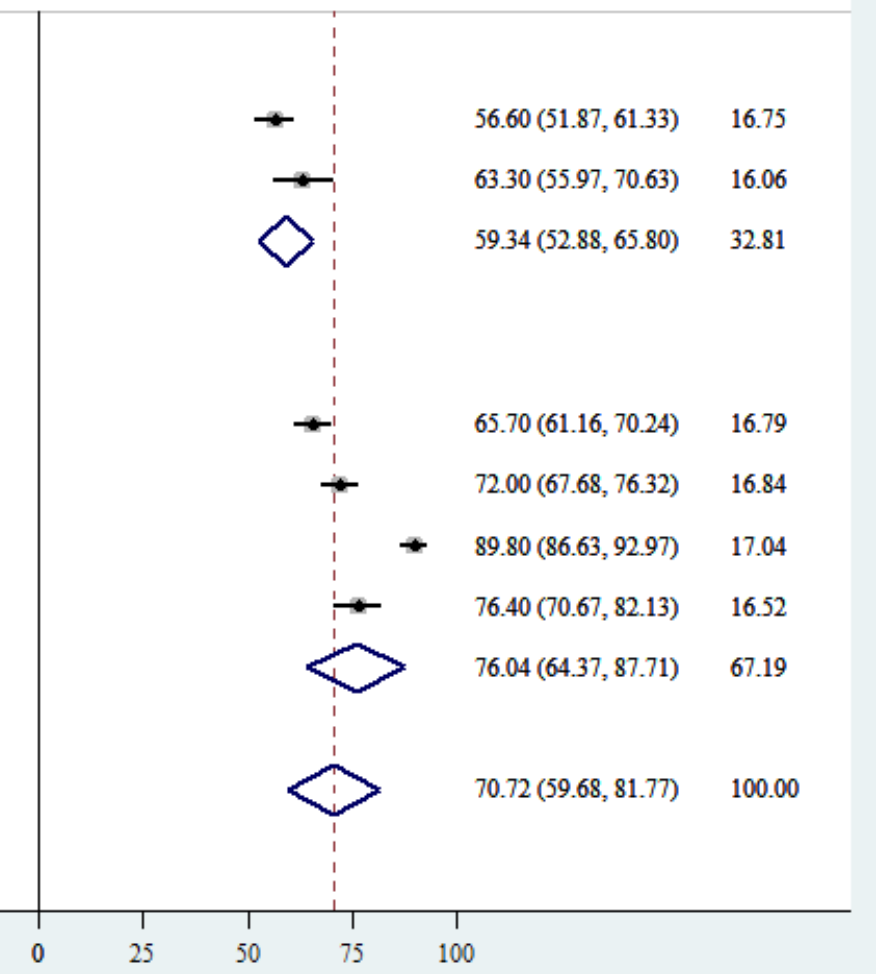

Figure 7. Sub-group analysis of attitude by publication status. 


\section{$\geq 347$}

Abay Woday Tadesse et al (2020)

Bedru Jemal et al (2020)

Belayneh Ayanaw Kassie et al (2020)

Degena Bahrey Tadesse et al (2020)

Mulusew Andualem et al (2020)

Henok Dagne et al (2020)

Yared Asmare Aynalem et al (2020)

Subtotal (I-squared $=96.2 \%, \mathrm{p}=0.000$ )

$<347$

Dejen Getaneh Feleke et al (2020)

Zelalem Tilahun et al (2020)

Hailemariam Gezie et al (2019)

Subtotal (I-squared $=98.1 \%, \mathrm{p}=0.000$ )

Overall (I-squared $=96.6 \%, \mathrm{p}=0.000)$

NOTE: Weights are from random effects analysis

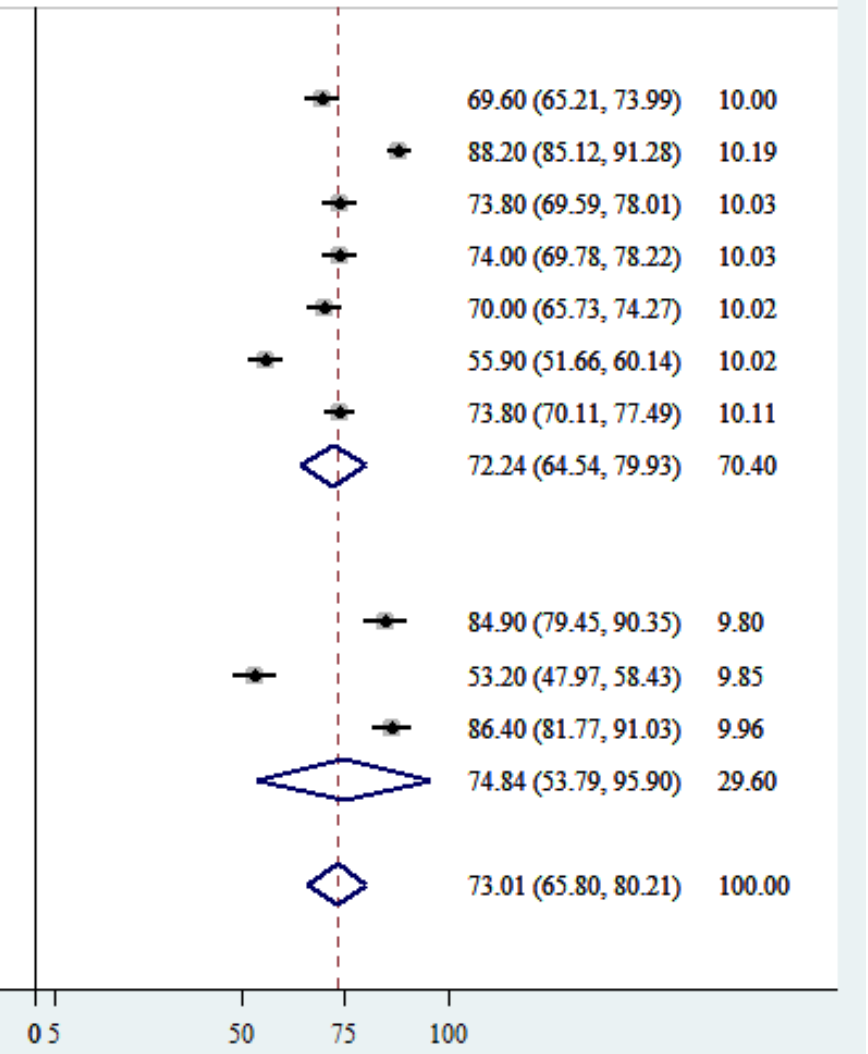

Figure 8. Sub-group analysis of knowledge by sample size.
First author with study year

$\geq 347$

Abay Woday Tadesse et al (2020)

Belayneh Ayanaw Kassie et al (2020)

Degena Bahrey Tadesse et al (2020)

Subtotal (I-squared $=91.0 \%, \mathrm{p}=0.000)$

$<347$

Dejen Getaneh Feleke et al (2020)

Zelalem Tilahun et al (2020)

Hailemariam Gezie et al (2019)

Subtotal (I-squared $=96.0 \%, \mathrm{p}=0.000$ )

Overall (I-squared $=97.1 \%, p=0.000$ )

NOTE: Weights are from random effects analysis
Attitude $95 \%$ CI $\quad \%$

$56.60(51.87,61.33) \quad 16.75$

$65.70(61.16,70.24) \quad 16.79$

$72.00(67.68,76.32) \quad 16.84$

$64.81(56.09,73.53) \quad 50.38$

$63.30(55.97,70.63) \quad 16.06$

17.04

16.52

49.62

100.00

Figure 9. Sub-group analysis of attitude by sample size. 
Publication Bias: Egger's test for small-study effects was performed to see the existence of publication bias. However, this test indicated that there was no publication bias with $\mathrm{P}=0.201$ for knowledge and $\mathrm{P}=0.144$. Moreover, the funnel plot also indicated that there was no publication bias suggesting that publication bias was not the cause for heterogeneity. Though funnel plot is usually interpreted subjectively, it is still a good tool to provide evidence. The existence of publication bias is usually evident if plots are fall symmetrically to the mean effect size within the funnel plot (Figures $10 \& 11$ ).

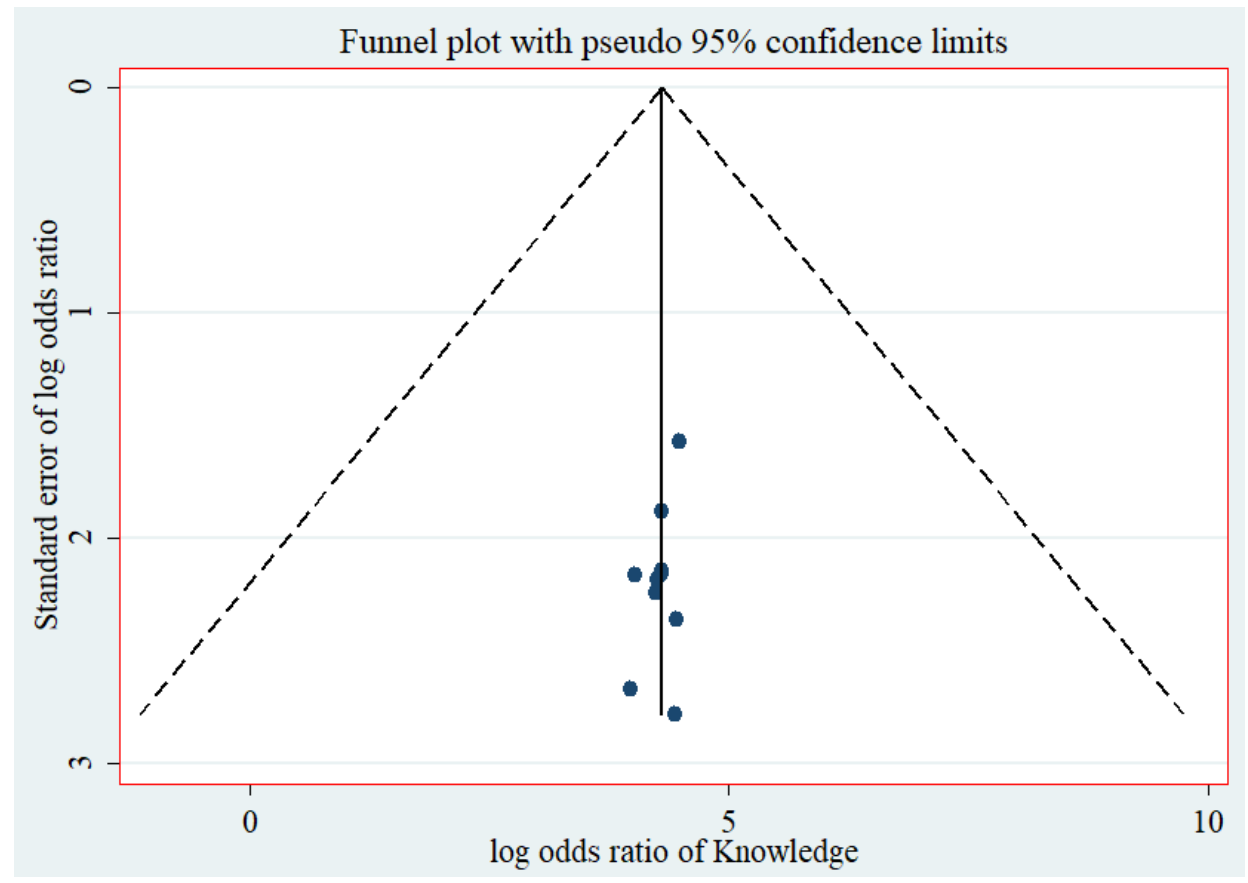

Figure 10. Funnel plot, in which the vertical line indicates the effect size whereas the diagonal line indicates the precision of individual studies with $95 \%$ confidence limit.

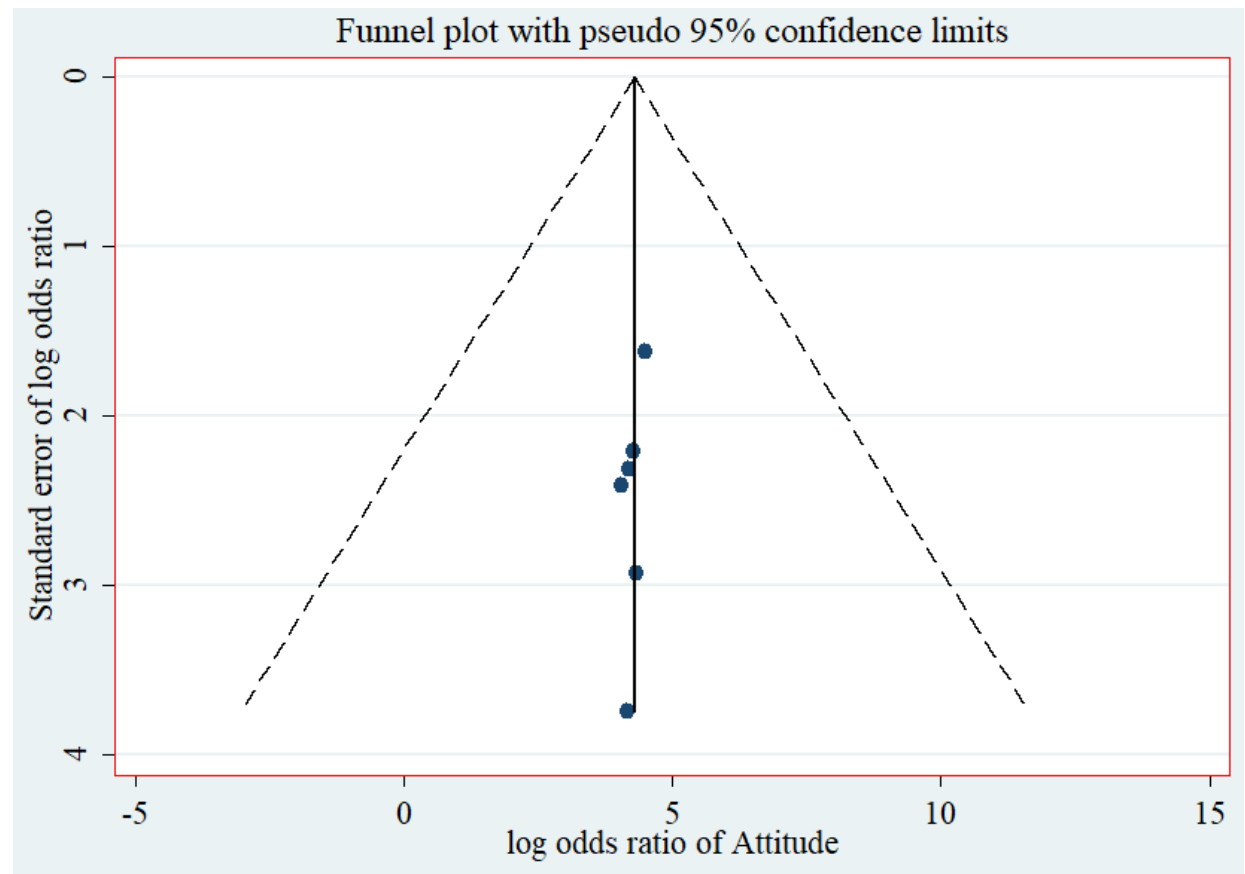

Figure 11. Funnel plot, in which the vertical line indicates the effect size whereas the diagonal line indicates the precision of individual studies with 95\% confidence limit.

Meta regression: meta-regression was further performed to find out cause of heterogeneity against region, year of study and sample size. None of the variables had a significant association with high level of heterogeneity for both knowledge and attitude towards COVID-19 (Table 2 and 3). 
Table 2. Meta-regression output for assessing causes of heterogeneity among studies included in knowledge.

\begin{tabular}{lllll}
\hline Variable & Coef & $\mathbf{P}>/ \mathbf{t} /$ & $\mathbf{9 5 \% C I}$ & \\
\hline \multirow{2}{*}{ Region } & -8.400084 & 0.297 & -25.76138 & 8.961209 \\
Publication status & Ref. & Ref. & Ref. & Ref. \\
Sample size & -2.959916 & 0.716 & -21.07083 & 15.151 \\
\hline
\end{tabular}

Table 3. Meta-regression output for assessing causes of heterogeneity among studies included in attitude.

\begin{tabular}{lllll}
\hline Variable & Coef & P>/t/ & $\mathbf{9 5 \% C I}$ & \\
\hline \multirow{2}{*}{ Region } & 15.54566 & 0.133 & -7.398351 & 38.48967 \\
Publication status & Ref. & Ref. & Ref. & Ref. \\
Sample size & 16.27638 & 0.119 & -6.576025 & 39.12879 \\
\hline
\end{tabular}

\subsection{Factors Affecting Healthcare Providers' Knowledge Towards COVID 19}

Originally, the odds of having adequate knowledge towards COVID-19 was higher among healthcare providers working in health facilities situating in urban areas compared to those health facilities situating in rural areas $[\mathrm{AOR}=0.44$,
95\% CI (0.26-0.70)] [20], and $[\mathrm{AOR}=4.3,95 \%$ CI $(2.6-$ 15.8)] [22]. Consistently, the pooled effect also revealed that the odds of having adequate knowledge towards COVID 19 was $76 \%$ less likely among healthcare providers working in health facilities of rural areas compared to those health facilities of urban areas $[\mathrm{AOR}=0.24,95 \% \mathrm{CI}(0.14-0.43)$ ] (Figure 12).

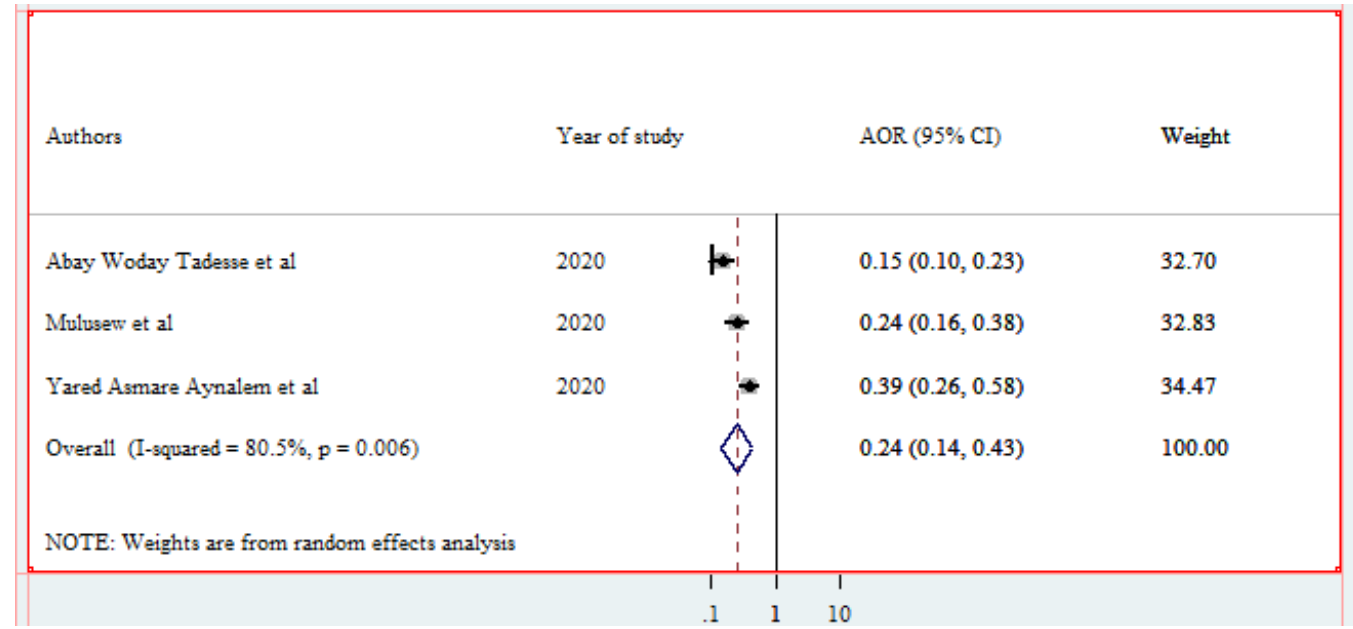

Figure 12. Forest plot of the adjusted odds ratios (AOR) with corresponding $95 \%$ CIs of studies on the association of residence and knowledge towards COVID 19.

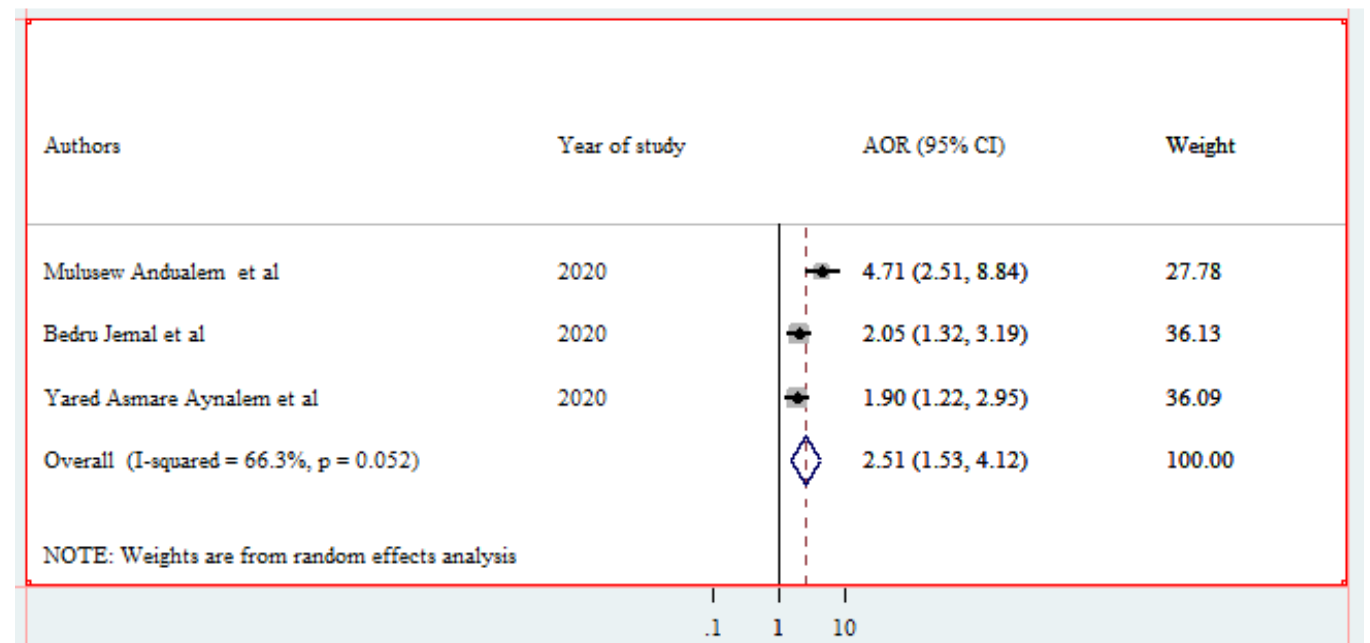

Figure 13. Forest plot of the adjusted odds ratios (AOR) with corresponding $95 \%$ CIs of studies on the association of social media use and knowledge towards COVID 19 . 


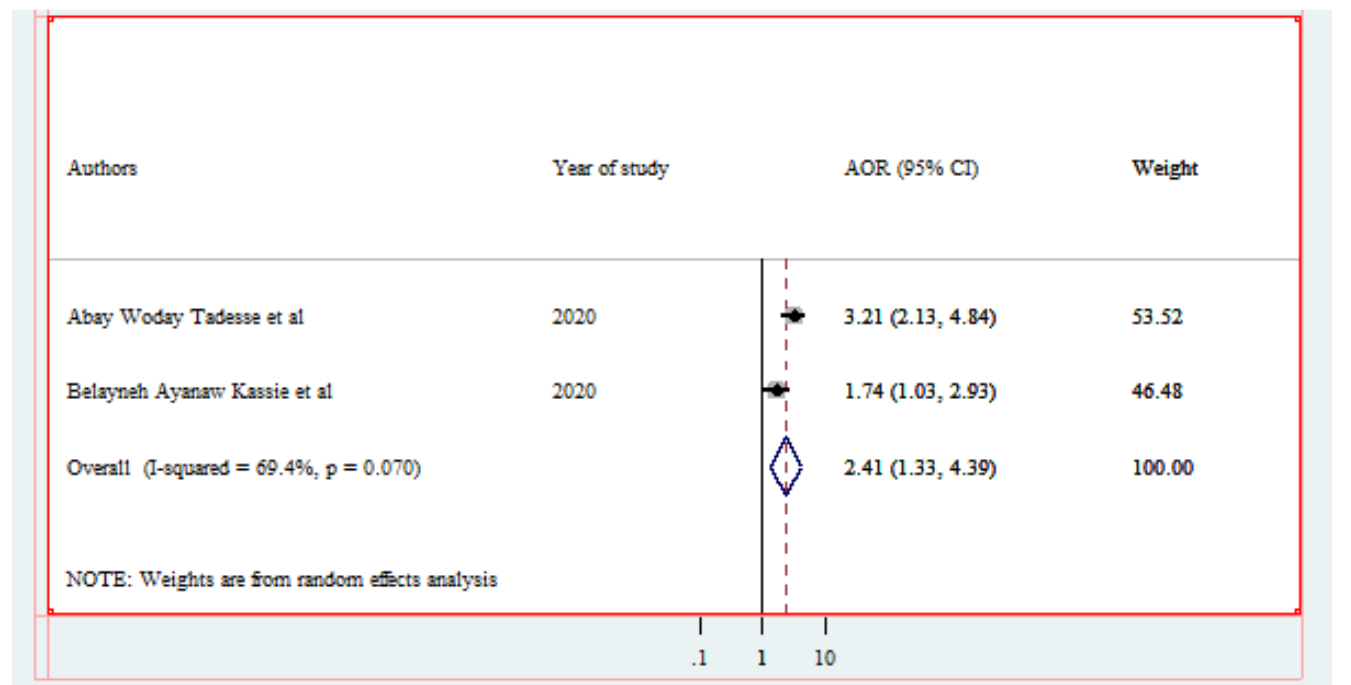

Figure 14. Forest plot of the adjusted odds ratios (AOR) with corresponding $95 \%$ CIs of studies on the association of educational status and attitude towards COVID 19 .

Concerning the source of information, the research findings also showed that those healthcare providers who were getting information regarding COVID 19 from social media were more likely to have adequate knowledge towards COVID 19 compared to their counterparts [AOR $=$ $2.51,95 \% \mathrm{CI}(1.42-4.53)]$ [20], and $[\mathrm{AOR}=3.41,95 \% \mathrm{CI}$ (1.56-7.43)] [26]. The pooled effect size also indicated that those healthcare providers who were getting information regarding COVID 19 from social media were 2.51 times more likely to have adequate knowledge of COVID 19 compared to their counterparts $[\mathrm{AOR}=2.51$, 95\% CI (1.53-4.12)] (Figure 13).

\subsection{Factors Affecting Healthcare Providers' Attitude Towards COVID 19}

Two research articles included in the review indicated that those healthcare providers with educational status of bachelor degree or above were more likely to have favorable attitude towards COVID 19 compared to those healthcare providers with educational status of diploma $[\mathrm{AOR}=0.27,95 \% \mathrm{CI}$ $(0.17-0.42)]$ [19], and [AOR=1.74, 95\% CI (1.034-2.928)] [21]. The pooled estimate also revealed that those healthcare providers with educational status of bachelor degree or above were 2.41 times more likely to have favorable attitude towards COVID 19 compared to those healthcare providers with educational status of diploma $[\mathrm{AOR}=2.41,95 \% \mathrm{CI}$ (1.33-4.39)] (Figure 14).

It was also found that those health care providers with adequate knowledge regarding COVID 19 had a favorable attitude towards COVID 19 as well compared to those with unfavorable knowledge towards COVID $19[\mathrm{AOR}=0.31$, 95\% CI (0.19-0.48)] [19], and [AOR $=3.17,95 \%$ CI $(1.97-$ 5.06)] [21]. Similarly, the pooled estimate suggests that health care providers with adequate knowledge regarding COVID 19 were 3.06 times more likely to have a favorable attitude towards COVID 19 compared to those with unfavorable knowledge towards COVID $19[\mathrm{AOR}=3.06$, 95\% CI (2.23-4.19)] [21] (Figure 15).

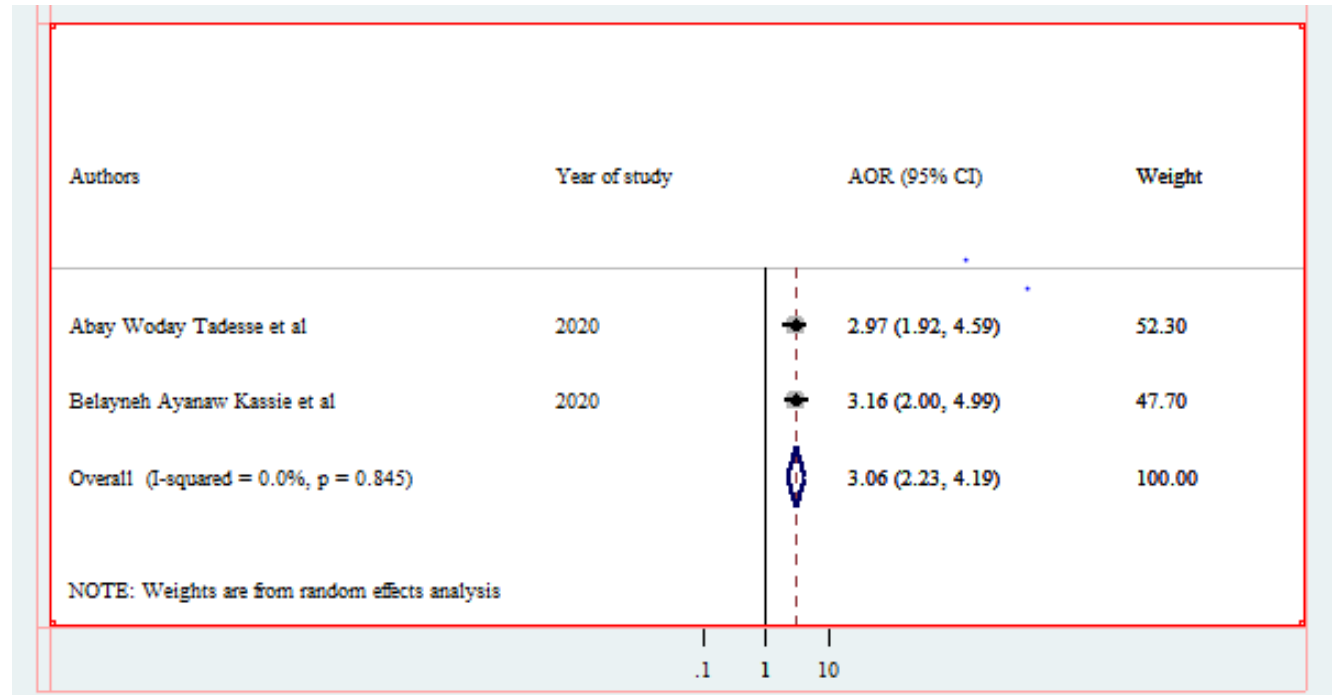

Figure 15. Forest plot of the adjusted odds ratios (AOR) with corresponding 95\% CIs of studies on the association of knowledge and attitude towards COVID 19. 


\section{Discussion}

Worldwide, the burden of COVID 19 in terms of treating patients and educating the public at large rests on the shoulder of healthcare providers. Logically, healthcare providers are usually expected to have adequate knowledge and a favorable attitude towards COVID 19. So, in Ethiopia researches has been conducted to see healthcare providers' knowledge and attitude towards COVID 19. However, inconsistent findings ranging from $53.2 \%$ to $88.2 \%$ for knowledge [17-26] and from $56.6 \%$ to $89.8 \%$ for attitude [17, 19, 21, 23-26] were recorded. Therefore, the pooled estimate would make decision-making easier for healthcare administrators and other stakeholders.

Consequently, the pooled prevalence of knowledge towards COVID-19 among healthcare providers in Ethiopia was $73.01 \%$. This finding is lower than the findings from China [8], India [10], Greece [32], but higher than the other findings from Bangladesh [33], India [11], Iran [34], Pakistan [35], Pakistan [36], Ghana [15], Uganda [16], and Nigeria [37]. Inconsistency was also observed with the pooled prevalence of attitude towards COVID-19 among healthcare providers in Ethiopia which was $70.72 \%$. It was lower than the findings from China [8], Vietnam [9], India [10], Yemen [14], Pakistan [36], and Nigeria [37] but higher than the findings from Ghana [15], and Uganda [16]. The possible explanation for inconsistencies could be related to differences in tools used to measure knowledge and attitude, study setting, and period.

Regarding Knowledge, the pooled estimates of this review indicated that the odds of having adequate knowledge towards COVID 19 were $76 \%$ less likely among healthcare providers working in health facilities of rural Ethiopia compared to those working in health facilities of urban Ethiopia. This was supported by a finding from Bangladesh [33]. In fact, the finding from Bangladesh [33] was conducted among internet users.

A positive association was also obtained regarding the source of information about COVID 19. Hence, healthcare providers who were using social media as a source of information were 2.51 times more likely to have adequate knowledge of COVID 19 compared to their counterparts. Similar evidence was observed in Uganda [16].

Healthcare providers who owned bachelor's degrees or above in their academics were 2.41 times more likely to have a favorable attitude towards COVID 19 compared to diploma holder healthcare providers in Ethiopia. Evidence from Yemen [14] suggested that there was a positive association between good educational status and good knowledge towards COVID 19.

Ethiopian healthcare providers who gained adequate knowledge of COVID 19 were 3.06 times more likely to develop a favorable attitude towards COVID 19 as well compared to those healthcare providers with inadequate knowledge of COVID 19. This was supported by evidence from Vietnam [9], Greece [32], and Nigeria [38].

Strengths and limitations of this review

1) The main strength of this review was providing the pooled effect size hence it could help researchers and policy makers to concentrate on finding solutions to the identified gaps.

2) Research articles included in this review operationally defined knowledge and attitude in somewhat differing ways therefore might have its own implication on the pooled effect size.

3) The pooled effect size reported in this review could change as time elapses because healthcare providers could learn about COVID 19 from their experience and trainings.

\section{Conclusion}

The level of knowledge and attitude towards COVID 19 among healthcare providers working in Ethiopia is very low compared to literatures reviewed and variables such as residence and social media use were factors affecting knowledge towards COVID 19 while educational status and knowledge were factors affecting attitude towards COVID 19 among healthcare providers.

\section{Data Availability}

The Excel data is available from the corresponding author upon request.

\section{Competing Interests}

The authors declare that they have no competing interests.

\section{Authors' Contributions}

Maru Meseret designed the study, prepared the protocol, supervised data collection, analyzed and interpreted the data.

Maru Meseret, Zegeye Regasa, Gizaw Hailye, and Bayou Tilahun coached the research from protocol development to data interpretation. Maru Meseret drafted and prepared the manuscript. All authors read and approved the final manuscript.

\section{Acknowledgements}

Authors, Maru Meseret, Zegeye Regasa, Gizaw Hailye, and Bayou Tilahun would like to thank HIT lab assistants for their willingness and motivation during data collection.

\section{References}

[1] Coronavirus disease 2019 (COVID-19) pandemic.: increased transmission in the EU/EEA and the UK - seventh update, 25 March 2020. Stockholm: ECDC. 2020; 31. 
[2] Schoeman D, Fielding BC. Coronavirus envelope protein: Current knowledge. Virol J 2019. 2019; 16 (1): 69.

[3] Li Q, et al. Early transmission dynamics in Wuhan, China, of novel coronavirus - infected pneumonia. New Engl J Medi.: 2001316.

[4] Yin Y, Wunderink RG: MERS, SARS and other coronaviruses as causes of pneumonia. Respirology; 2018; 23 (2): 130-137.

[5] ECDC. Information on COVID-19 for people with immunocompromising condition, their family members and care givers. 2020 .

[6] CDC. What you should know about COVID-19 to protect yourself and others. 2020 .

[7] WHO. Risk assessment and management of exposure of health care workers in the context of COVID-19; Interim guidance. 2020.

[8] Zhang M, et al. Knowledge, attitude, and practice regarding COVID-19 among healthcare workers in Henan, China. $J$ Hosp Infect [Internet]. 2020; 105 (2): 183-7. Available from: www.sciencedirect.com.

[9] Huynh Giao et al. Knowledge, Attitude, and Practices Regarding COVID-19 Among Chronic Illness Patients at Outpatient Departments in Ho Chi Minh City, Vietnam. Asian Pac J Trop Med. 2020; 13 (6): 260-5.

[10] Maheshwari S, Gupta PK, Sinha R, Rawat P. Knowledge, attitude, and practice towards coronavirus disease 2019 (COVID-19) among medical students: A cross-sectional study. $J$ Acute Dis 2020; 9 (3): 100-104.

[11] Paul et al. Assessment of knowledge gaps and perceptions about COVID-19 among health care workers and general public- national cross-sectional study. J Anaesthesiol Clin Pharmacol. 2020; 36 (3): 337-44. Available from: http://www.joacp.org

[12] Temsah MH et al. Knowledge, attitudes and practices of healthcare workers during the early COVID-19 pandemic in a main, academic tertiary care centre in Saudi Arabia. Epidemiol Infect. 2020; 148: 1-9.

[13] Bhagavathula et al. Knowledge and Perceptions of COVID-19 Among Health Care Workers. JMIR Public Heal Surveill. 2020; 6 (2): 1-9.

[14] Alrubaiee et al. preventive behaviours towards COVID-19 among health care providers in Yemen. BMC Public Health. 2020; 20: $1-11$.

[15] Dorcas Serwaa et al. Knowledge, risk perception and preparedness towards coronavirus disease-2019 (COVID-19) outbreak among Ghanaians. Pan Afr Med J. 2020; 35: 1-7.

[16] Olum R, Chekwech G WG, Nassozi DR and Bongomin F. Coronavirus Disease-2019: Knowledge, Attitude, and Practices of Health Care Workers at Makerere University Teaching Hospitals,. Public Health. 2020; 8: 181.

[17] Tesfaye ZT, Yismaw MB, Negash Z, Ayele AG. COVID-19Related Knowledge, Attitude and Practice Among Hospital and Community Pharmacists in Addis Ababa, Ethiopia. Integr Pharm Res Pract. 2020; 9.

[18] Henok Dagne et al: Prevention Practice and Associated Factors of Coronavirus disease 2019 (COVID-19) Outbreak among Educated Ethiopians: 2020.
[19] Tadesse A, Melese N, Eshetie S, Chane M, Ali A. Knowledge, Attitude, and Practice and Associated Factors towards COVID-19 among College Students in Amhara Region, Ethiopia. Res Sq. 2020.

[20] Asemahagn MA. Factors determining the knowledge and prevention practice of healthcare workers towards COVID-19 in Amhara region, Ethiopia. Trop Med Health. 2020; 48: 72.

[21] Kassie BA, Adane A, Tilahun YT, Kassahun EA, Ayele AS, Belew AK. Knowledge and attitude towards COVID-19 and associated factors among health care providers in Northwest Ethiopia. PLoS One. 2020; 15 (8): 1-12.

[22] Yared Asmare Aynalem, et al: Assessment of undergraduate student knowledge, practices, and attitude towards COVID-19 in Debre Berhan University, Ethiopia: 2020.

[23] Tadesse DB, Gebrewahd GT, Demoz GT. Knowledge, Attitude, Practice and Psychological response toward COVID19 among Nurses during the COVID-19 outbreak in Northern Ethiopia, 2020. Res Sq Prepr. 2020; 1-14.

[24] Getaneh D, Fetene S, Sisay E. Assessment of Knowledge, Attitude and Associated Factors toward COVID-19 among Nurses Who Work in South Gondar Zone, Hospitals, Northwest Ethiopia 2020. Osteoarthr Cartil. 2020; 28 (2): 1-27.

[25] Gezie H, Leta E, Admasu F, Gedamu S, Dires A, et al. Health care workers knowledge, attitude and practice towards hospital acquired infection prevention at Dessie referral hospital, Northeast Ethiopia. Clin J Nurs Care Pract. 2019; 3: 059-063.

[26] Bedru et al. Knowledge, attitude and practice of healthcare workers towards COVID-19 and its prevention in Ethiopia. Res Sq priprint. 2020; 1-14.

[27] Nguyen LH, Drew DA, Graham MS, Joshi AD, Guo CG, Ma W, et al. Risk of COVID-19 among front-line health-care workers and the general community. Lancet Public Heal. 2020; 5: 1-9.

[28] Sikkema RS, Pas SD, Nieuwenhuijse DF, O’Toole Á, Verweij J, van der Linden A, et al. COVID-19 in health-care workers in three hospitals in the south of the Netherlands. Lancet Infect Dis. 2020; 1-8.

[29] Hartmann et al. Coronavirus Disease 2019 (COVID-19) Infections Among Healthcare Workers, Los Angeles County, February-May 2020. Clin Infect Dis. 2020; 1-5.

[30] Lake EA, Demissie BW, Gebeyehu NA, Wassie AY, Gelaw KA, Azeze GA: Knowledge, attitude and practice towards COVID19 among health professionals in Ethiopia: A systematic review and meta-analysis: 2021: PLoS ONE 16 (2).

[31] The Joanna Briggs Institute: The Joanna Briggs Institute Critical Appraisal tools for use in JBI Systematic Reviews Checklist for Systematic Reviews and Research Syntheses: $2017 . \quad$ Available https://joannabriggs.org/sites/default/files/201905/JBI_Critical_AppraisalChecklist_for_Systematic_Reviews2017_0.pdf

[32] Dimitrios Papagiannis et al: Assessment of Knowledge, Attitudes, and Practices towards New Coronavirus (SARSCoV-2) of Health Care Professionals in Greece before the Outbreak Period: Int. J. Environ. Res. Public Health 2020. 
[33] Rahman A, Sathi NJ. Knowledge, Attitude, and Preventive Practices toward COVID-19 among Bangladeshi Internet Users. Electron J Gen Med. 2020; 17 (5).

[34] Marzieh Nemati, Bahareh Ebrahimi, and Fatemeh Nemati: Assessment of Iranian Nurses' Knowledge and Anxiety Toward COVID-19 During the Current Outbreak in Iran: Arch Clin Infect Dis: 2020.

[35] M. Saqlain et al.: Knowledge, attitude, practice and perceived barriers among healthcare workers regarding COVID-19: a cross-sectional survey from Pakistan: Journal of Hospital Infection: 2020.
[36] Usman Rashid Malik et al: Knowledge, Attitude, and Practices of Healthcare Professionals on COVID-19 and Risk Assessment to Prevent the Epidemic Spread: A Multicenter Cross-Sectional Study from Punjab, Pakistan: Int. J. Environ. Res. Public Health: 2020.

[37] Rine Christopher Reuben etal: Knowledge, Attitudes and Practices towards COVID-19: An Epidemiological Survey in North-Central Nigeria: Journal of Community Health: 2020.

[38] Okoro J, Ekeroku A, Nweze B et al. Attitude and preventive practices towards COVID-19 disease and the impact of awareness training on knowledge of the disease among correctional officers: Emerald Open Research: 2020, 2: 51. 